\title{
Boosting immunity to Omicron
}

\author{
New data confirm that the COVID-19 vaccine booster dose is crucial for the generation of neutralizing antibody \\ responses against Omicron, while better therapeutic antibodies are needed for this and future variants.
}

\author{
Xiaoying Shen
}

\begin{abstract}
n December 2021, the SARS-CoV-2 Omicron variant swiftly overtook the Delta variant to become globally dominant in the COVID-19 pandemic. Omicron contains a large number of mutations $(>30)$ in the spike protein, including 17 within the receptor binding domain (RBD), the major target for neutralizing antibodies. Epidemiology data on Omicron-associated reinfection and vaccine breakthrough infections ${ }^{1}$ suggest serious immune evasion by this variant. Understanding the scope and extent of resistance of Omicron to vaccine-elicited immune responses, especially following a COVID-19 vaccine booster, is crucial for future vaccine development and public health policies.
\end{abstract}

In this issue of Nature Medicine, three studies by Pérez-Then et al. ${ }^{2}$, Cheng et al. ${ }^{3}$ and Gruell et al. ${ }^{4}$ present data on the neutralizing antibody response against Omicron induced by vaccine regimens with and without a booster immunization, and a fourth study by VanBlargan et al..$^{5}$ reports on the susceptibility of Omicron to therapeutic antibodies. These four studies, together with recent real-world reports of vaccine efficacy against Omicron, begin to illustrate the threat of this variant to current vaccines and monoclonal antibody $(\mathrm{mAb})$ therapies, and suggest ways to mitigate this threat.

The Omicron variant contains mutations that confer resistance to at least four classes of mAbs targeting the RBD of the spike protein $^{6,7}$. The studies by VanBlargan et al. ${ }^{5}$ and Gruell et al. ${ }^{4}$ showed extensive evasion by Omicron of neutralization by several $\mathrm{mAbs}$ that are used therapeutically to treat infection. Omicron was highly resistant to neutralization by more than onehalf of the mAbs tested, including bamlanivimab (also known as LY-CoV555), etesevimab (LV-CoV016), REGN10933, REGN10987, CT-P59, C102, P2B-2F6 and Fab2-36. Fortunately, not all therapeutic antibodies lost their activity against Omicron. Neutralization potency of Sotrovimab (S309) was minimally affected; the investigational antibody DZIF-10c and the investigational LY-CoV2-2196/COV2-2130 combination

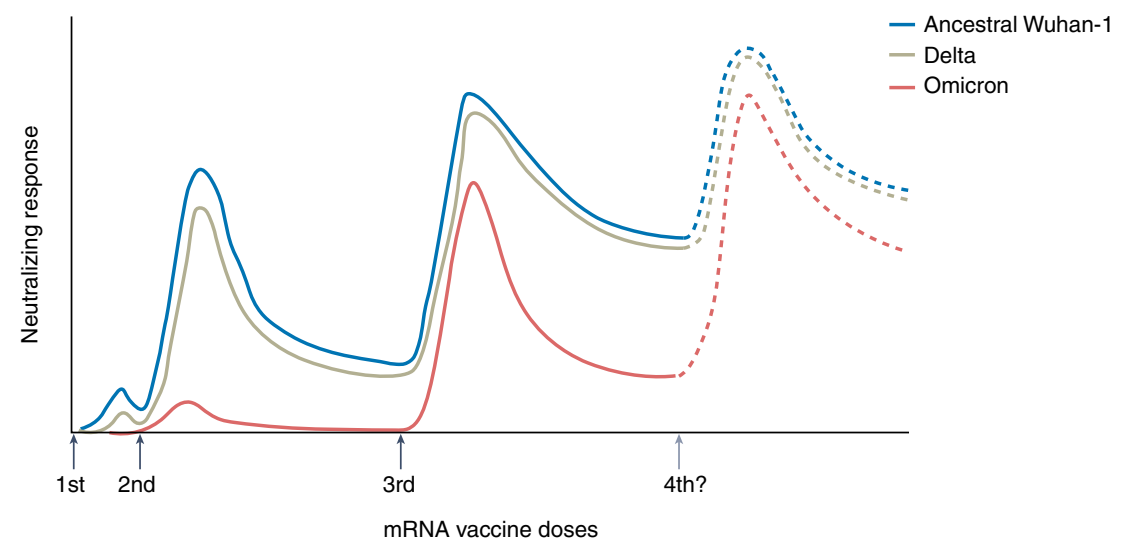

Fig. 1 | A booster dose is crucial for neutralizing antibody responses against Omicron. After two mRNA vaccine doses, there is minimal antibody-medicated neutralization of Omicron. Robust neutralization of Omicron develops after a third mRNA vaccine dose. The magnitude of Omicron neutralization after a third (booster) dose is comparable to neutralization of Delta after two doses. A fourth dose (second booster) has the potential to further improve the magnitude and durability of the immune response. Dashed lines indicate hypothetical response levels.

also retained Omicron neutralization activity, albeit with 12-25-fold reduced potency. These findings highlight the need for development of better therapeutic mAbs to effectively counter emergent variants that may be even more resistant. Research in this field will also help regulatory authorities and healthcare providers to choose the appropriate $\mathrm{mAb}$ treatments for Omicron infections.

Three studies evaluated the extent to which Omicron evades vaccine-elicited neutralizing antibodies, highlighting the importance of a booster shot. Pérez-Then et al. ${ }^{2}$ studied vaccine recipients in the Dominican Republic who received two doses of CoronaVac (inactivated whole virus vaccine, Sinovac) followed by a BNT162b2 (mRNA vaccine, Pfizer) booster. Cheng et $\mathrm{al}^{3}{ }^{3}$ evaluated vaccine recipients in China who received two doses of CoronaVac followed by a CoronaVac or a BNT162b2 booster and finally, Gruell et al. ${ }^{4}$ evaluated vaccine recipients in Germany who received two or three doses of the BNT162b2 vaccine. The three studies engaged different study cohorts, included different vaccine regimens, and utilized different neutralization assay platforms (live virus and pseudovirus assays). Nevertheless, all three studies showed clearly that Omicron neutralization is weak or undetectable after two doses of mRNA or inactivated vaccine, and a booster immunization is crucial for a strong neutralizing antibody response against Omicron.

Responses against both the ancestral Wuhan-1 virus, which is used in the vaccines, and the Omicron variant, were considerably lower for the CoronaVac vaccine, whether administered as part of a primary immunization series or as a booster, than for the BNT162b2 mRNA vaccine $^{2,3}$. Among people who received two vaccine doses, neutralizing antibodies against Omicron were detected in just $30-37 \%$ of people who received BNT162b2 and $0 \%$ of people who received CoronaVac. Crucially, however, a BNT162b2 booster after either regimen increased the response rate against Omicron - to $100 \%$ if given after BNT162b2 primary immunization ${ }^{3,4}$ and to more than $90 \%$ if given after CoronaVac ${ }^{3}$. The levels of neutralizing 
antibodies against Omicron were improved 7- to 100-fold with an mRNA booster, so that they were ultimately within 2 -fold of the level of response against ancestral Wuhan-1 in all three studies, which is consistent with other reports ${ }^{8}$. Because neutralizing antibody levels have tended to correlate with COVID-19 vaccine-mediated protection ${ }^{9}$, it is reasonable to expect similar levels of protection against Omicron after an mRNA booster to that seen against earlier variants after two vaccine doses. These findings also raise the question of whether an additional boost will be needed to further improve the response against Omicron for optimal durable protection (Fig. 1).

These findings are in agreement with recent vaccine efficacy data for Omicron and Delta in three large-scale studies from the US Centers for Disease Control and Prevention (CDC) $)^{10-12}$ showing higher vaccine efficacy against both Delta and Omicron after an mRNA vaccine booster. In particular, three doses of mRNA vaccine showed 94\% and $90 \%$ efficacy at preventing COVID19-associated hospitalizations during the Delta- and Omicron-predominant phases of the pandemic, respectively. By contrast, within 6 months of the second dose, vaccine efficacy against COVID19-associated hospitalizations were 90\% and $81 \%$, respectively ${ }^{11}$. Protection against symptomatic infection is also improved after the booster immunization; the adjusted odds ratio for three versus two mRNA vaccine doses was 0.16 for Delta and 0.34 for Omicron, respectively, indicating a lesser likelihood of symptomatic infection with three versus two doses ${ }^{12}$.
One caveat of the above-mentioned vaccine efficacy studies is that only data collected over a relatively short period of time after the booster dose were available, so they do not provide information on durability of the response. Previous studies show that vaccine efficacy after the second mRNA immunization was lower at 6 months or later, compared to within the first 6 months ${ }^{11}$. Durability of the immune response against Omicron, especially after the booster dose, is an important consideration when deciding if and when an additional booster is needed. A recent analysis of the durability of antibody responses following mRNA-1273 primary and booster immunizations ${ }^{13}$ revealed that neutralizing antibodies against the prototype D614G variant - which was dominant at the time when the Moderna and Pfizer vaccines showed outstanding efficacy that led to their Emergency Use Authorizations - declined 7.8-fold from 1 month to 7 months after the second mRNA immunization, whereas the decline from 1 month to 6 months after the booster was only 2.3 -fold. This suggests improved durability after the boost; however, the response against Omicron declined 6.3-fold over the same 6-month time frame after the boost. It will therefore be important to determine whether an additional boost will bring about a more durable response against Omicron.

Vaccines that specifically target Omicron are currently being tested as boosters, and as SARS-CoV-2 continues to evolve, vaccines that can elicit broad cross-variant neutralizing responses are highly desirable. Vaccine designs focusing on broadly neutralizing antibodies are in early stages of development, including pan-coronavirus vaccines that aim to target future SARS-CoV-2 variants as well as other sarbecoviruses that may jump to humans in the future ${ }^{14,15}$. These avenues of research warrant investment of resources now and into the future.

\section{Xiaoying Shen (1D)}

Department of Surgery and Human Vaccine Institute, Duke University Medical Center, Durham, NC, USA.

$凶_{e-m a i l: s x s h e n @ d u k e . e d u}$

Published online: 24 February 2022

https://doi.org/10.1038/s41591-022-01727-0

References

1. Kuhlmann, C. et al. Lancet https://doi.org/10.1016/S01406736(22)00090-3 (2022).

2. Pérez-Then, E. et al. Nat. Med. https://doi.org/10.1038/s41591022-01705-6 (2022).

3. Cheng, S. M. S. et al. Nat. Med. https://doi.org/10.1038/s41591022-01704-7 (2022).

4. Gruell, H. et al. Nat. Med. https://doi.org/10.1038/s41591-02101676-0 (2022).

5. VanBlargan, L. A. et al. Nat. Med. https://doi.org/10.1038/s41591021-01678-y (2022).

6. Liu, L. et al. Nature https://doi.org/10.1038/s41586-021-04388-0 (2021).

7. Hastie, K. M. et al. Science 374, 472-478 (2021).

8. Muik, A. et al. Science https://doi.org/10.1126/science.abn7591 (2022).

9. Gilbert, P. B. et al. Science 375, 43-50 (2022).

10. Johnson, A. G. et al. Morb. Mortal. Wkly Rep. https://doi. org/10.15585/mmwr.mm7104e2 (2022).

11. Thompson, M. G. et al. Morb. Mortal. Wkly Rep. https://doi. org/10.15585/mmwr.mm7104e3 (2022).

12. Accorsi, E. K. et al. JAMA https://doi.org/10.1001/jama.2022.0470 (2022).

13. Pajon, R. et al. N. Engl. J. Med. https://doi.org/10.1056/ NEJMc2119912 (2022).

14. Saunders, K. O. et al. Nature 594, 553-559 (2021).

15. Cohen, A. A. et al. Science 371, 735-741 (2021).

The author declares no competing interests.

\section{Polygenic embryo testing: understated ethics, unclear utility}

New technologies are expanding the reach and accessibility of preimplantation genetic testing of human embryos. But what these advances can deliver is still unclear, and a frank assessment of their profound ethical implications is urgently needed.

\section{Josephine Johnston and Lucas J. Matthews}

$\mathrm{n}$ fertility medicine, preimplantation genetic testing (PGT) has been developed for two purposes: first, to improve in vitro fertilization (IVF) birth rates by assessing embryo viability; and second, to enable prospective parents to transfer for gestation only those embryos that do not carry specific rare disease genes. In 2019, just $2.1 \%$ of babies born in the United States were conceived by IVF, and only a small number of parents, mainly those with a family history of genetic conditions such as 